\title{
Neurodesenvolvimento de Grandes Prematuros ou Recém-Nascidos com Muito Baixo Peso: Comparação de Gémeos Monocoriónicos e Bicoriónicos com Recém-Nascidos de Gestação Unifetal
}

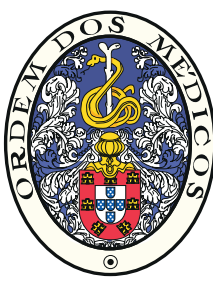

\author{
Neurodevelopmental Outcomes of Very Preterm \\ or Very Low Birth Weigth Infants: Comparison of \\ Monochorionic and Dichorionic Twins with Singletons
}

\author{
Adelaide TABORDA $\triangle^{1}$, Guiomar OLIVEIRA ${ }^{2,3}$ \\ Acta Med Port 2016 Nov;29(11):702-710 - http://dx.doi.org/10.20344/amp.7079
}

\section{RESUMO}

Introdução: Estudos evidenciaram maior taxa de alterações do neurodesenvolvimento nos gémeos em relação aos recém-nascidos de gestação unifetal. O objetivo deste trabalho foi comparar alterações do neurodesenvolvimento em gémeos (monocoriónicos e bicoriónicos) grandes prematuros ou de muito baixo peso ao nascer, com recém-nascidos de gestação unifetal.

Material e Métodos: Estudo retrospetivo de uma coorte de recém-nascidos com idade gestacional inferior a 32 semanas ou peso de nascimento inferior a $1500 \mathrm{~g}$, internados na Unidade de Cuidados Intensivos Neonatais, numa maternidade de apoio perinatal diferenciado da Região Centro de Portugal, no período de 2006 a 2010. A avaliação do neurodesenvolvimento foi realizada aos 24 meses, com a escala de Growing Skills II. No diagnóstico de paralisia cerebral usou-se a classificação internacional de Surveillance of Cerebral Palsy in Europe. Foram comparados recém-nascidos de gestação unifetal com recém-nascidos de gravidez múltipla e com os subgrupos: monocoriónicos e bicoriónicos. Análise estatística pelo SPSS versão 20.0. Foi aplicado um modelo de regressão logística. Resultados: Foram avaliados 194 recém-nascidos do grupo gestação unifetal e 89 gémeos - 50 bicoriónicos e 39 monocoriónicos. Os gémeos monocoriónicos apresentaram maior risco, em relação ao grupo de gestação unifetal, de alterações moderadas a graves do neurodesenvolvimento global (OR ajustado 3,6) e nas subáreas: locomoção (OR ajustado 12,2) linguagem (OR ajustado 6,5) e autonomia (OR ajustado 7,2). A paralisia cerebral foi diagnosticada em 15,4\% dos gémeos monocoriónicos e $4,1 \%$ de gestação unifetal (OR ajustado 4,2).

Discussão: Este trabalho evidenciou taxa superior de alterações moderadas a graves do neurodesenvolvimento, incluindo a paralisia cerebral nos gémeos monocoriónicos em relação ao grupo de gestação unifetal. A análise por grupos estratificados em relação à idade gestacional e a comparação de monocoriónicos com bicoriónicos alertou para o papel da corionicidade na base destas sequelas.

Conclusão: Podemos concluir que os gémeos monocoriónicos apresentaram, na população estudada, um risco significativo de sequelas moderadas a graves do neurodesenvolvimento.

Palavras-chave: Córion; Deficiências do Desenvolvimento; Gémeos; Paralisia Cerebral; Recém-Nascido Extremamente Prematuro; Recém-Nascido de Muito Baixo Peso.

\section{ABSTRACT}

Introduction: Twins are associated with a delayed development and cerebral palsy. The purpose of this work was to compare the neurologic morbidity in very preterm or very low birth weight dichorionic and monochorionic twins with singletons.

Material and Methods: We conducted a retrospective cohort study of livebirths lowest through 32 weeks of gestation or very low weight infants admitted to Neonatal Intensive Care Unit of a level III hospital, between 2006 and 2010. Development was evaluated with the Growing Skills II Scale at 24 months of age. Cerebral palsy was defined by predetermined criteria by Surveillance of Cerebral Palsy in Europe. Infants were analyzed as twins and singletons cohort. Within the twin category the infants were further separated as dichorionic and monochorionic and were compared with singletons infants. Logistic regression models were used to control for demographic and clinical factors.

Results: The cohort of infants who were assessed for neurodevelopmental impaired, consisted of 194 singletons infants and 89 twins (50 dichorionic; 39 monochorionic). Monochorionic twins, when compared with the singletons, showed an increased risk of severe developmental delay in these areas: locomotion (adjusted OR 12.2) language (adjusted OR 6.5) and autonomy (adjusted OR 7.2). Cerebral palsy was diagnosed in $4.1 \%$ of singleton infants and $15.4 \%$ of monochorionic twins. The adjusted risk of severe developmental delay and cerebral palsy in monochorionic twins was 3.6 and 4.2 , respectively.

Discussion: This work showed higher rate of moderate and severe neurodevelopment delay including cerebral palsy in monochorionic twins compared to singletons infants. Analysis by groups stratified according to gestational age and comparison of monochorionic and dichorionic twins displayed the role of chorionicity for these neurodevelopmental disorders.

Conclusions: In our sample the monochorionic twins are associated with an independent risk of neurodevelopmental delay. Keywords: Cerebral Palsy; Chorion; Developmental Disabilities; Infant, Extremely Premature; Infant, Very Low Birth Weight; Twins.

1. Serviço de Neonatologia. Maternidade Bissaya Barreto. Centro Hospitalar e Universitário de Coimbra. Coimbra. Portugal.

2. Unidade de Neurodesenvolvimento e Autismo. Serviço do Centro de Desenvolvimento da Criança e Centro de Investigação e Formação Clínica. Hospital Pediátrico. Centro

Hospitalar e Universitário de Coimbra. Coimbra. Portugal.

3. Clínica Universitária de Pediatria e Instituto de Imagem Biomédica e Ciências da Vida. Faculdade de Medicina. Universidade de Coimbra. Coimbra. Portugal.

$\square$ Autor correspondente: Adelaide Taborda: adelaide.taborda@gmail.com

Recebido: 10 de dezembro de 2015 - Aceite: 07 de março de 2016 | Copyright @ Ordem dos Médicos 2016 


\section{INTRODUÇÃO}

Nas últimas duas décadas, tem-se assistido ao aumento do número de gestações múltiplas, em parte explicado pelo crescente recurso a técnicas de fertilização e gravidez medicamente assistida. Este tipo de gestação está associado a um incremento da frequência de prematuridade e de baixo peso ao nascer. ${ }^{1,2}$

Vários trabalhos, incluindo estudos populacionais, têm revelado taxas superiores de mortalidade e morbilidade, ${ }^{2-5}$ nomeadamente na área do neurodesenvolvimento nos recém-nascidos (RN) de gestação múltipla (GM) relativamente aos de gestação unifetal (GUF).

A gemelaridade tem sido referida como um fator de risco para a paralisia cerebral (PC). Estima-se que a PC, a nível europeu, apresente uma incidência de 2,1 por mil nados vivos, ${ }^{6}$ variando com a idade gestacional (IG). Na verdade, estima-se, por mil nascimentos, 146 casos de PC abaixo das 28 semanas de IG; 62 entre as 28 e 31 semanas; sete entre as 32 e as 36 e 1,1 nos RN de termo. ${ }^{7,8}$

Foram referidas taxas de PC cinco a dez vezes superiores em crianças nascidas de $\mathrm{GM}$, relativamente às que provieram de GUF. 4,9

As elevadas taxas de mortalidade, morbilidade e de evolução neurológica desfavorável a longo prazo em gémeos tem sido explicada pela associação a baixa IG e a menor peso de nascimento. Contudo, a contribuição relativa de fatores como a prematuridade e a gemelaridade, no prognóstico, é difícil de distinguir. Há trabalhos que atribuem à gemelaridade, sobretudo se for monocoriónica (MC), a causa da frequência acrescida de alterações do neurodesenvolvimento, nomeadamente de PC.5,10,11

Está descrito que o gémeo sobrevivente de uma gravidez com um feto morto tem elevado risco de PC e outras alterações do neurodesenvolvimento, ${ }^{4,9,12}$ sendo este risco significativamente superior se a gravidez múltipla (GM) for MC.

As alterações neurológicas no gémeo sobrevivente podem ser explicadas pela passagem de material trombótico do feto morto para o saudável e pelas alterações hemodinâmicas resultantes de transfusão de sangue do feto vivo para o morto, gerando períodos de hipoperfusão, hipotensão e anemia fetal aguda. ${ }^{5,9,10}$

A síndrome de transfusão feto fetal (STFF), complicação da gravidez múltipla monocoriónica (GM-MC), constitui também um fator de risco elevado para o aparecimento de alterações neurológicas. ${ }^{9,13}$

Vários estudos têm procurado comparar as alterações neurológicas ou de neurodesenvolvimento estratificando os gémeos por peso de nascimento, mas os resultados têm sido contraditórios. ${ }^{3,13,14}$ Não há muitos estudos que analisem estas alterações ajustando-as para a IG. Por outro lado, a corionicidade, também tem sido referida como responsável pela maior frequência das lesões cerebrais ou das alterações neurológicas que ocorrem nos gémeos, ${ }^{3,15}$ sendo mal conhecida em muitos estudos.

O objetivo deste trabalho foi comparar as alterações do neurodesenvolvimento em crianças grandes prematuras, nascidas de GUF versus GM e sobretudo avaliar a sua re- lação com a corionicidade, dividindo a $\mathrm{GM}$ em dois subgrupos: GM-MC versus gravidez múltipla bicoriónica (GM-BC).

A relevância de identificar os fatores que estão associados ao prognóstico neurológico de crianças nascidas de $\mathrm{GM}$, relaciona-se com a necessidade de informar e apoiar adequadamente as famílias, assim como conhecer indicadores de risco em que se possa antecipar a intervenção.

\section{MATERIAL E MÉTODOS}

Estudo observacional analítico retrospetivo de uma coorte de RN grandes prematuros (idade gestacional inferior a 32 semanas) e/ou de muito baixo peso ao nascer (peso inferior a $1500 \mathrm{~g}$ ), que estiveram internados na Unidade de Cuidados Intensivos Neonatais numa maternidade de apoio perinatal diferenciado da Região Centro de Portugal, Maternidade Bissaya Barreto (MBB), num período de cinco anos (1 de janeiro de 2006 a 31 de dezembro de 2010). Foi selecionada esta população uma vez que são realizados sistematicamente, a estes recém-nascidos, registos de dados da avaliação clínica até aos 2 - 3 anos $\mathrm{e}$, ainda, tendo em conta o facto de haver estudos que se reportam quer à IG quer ao $\mathrm{PN}$ como indicadores de prognóstico.

Foram excluídos dois RN com distrofia miotónica que não sobreviveram e um RN, com doença neurológica prévia, cuja patologia poderia interferir nas aquisições do neurodesenvolvimento.

Neste estudo, foram incluídos ambos os gémeos, mesmo que apenas um tivesse PN inferior a $1500 \mathrm{~g}$. As características perinatais dos RN e sua evolução, assim como o registo da corionicidade das gestações gemelares foram coletadas do registo escrito disponível no sistema informatizado de apoio ao médico (SAM), usado na Instituição. A informação relativamente à corionicidade foi obtida através dos relatos dos achados nas ecografias obstétricas realizadas por rotina, no primeiro trimestre de gestação, pelo Serviço de Obstetrícia da MBB.

A escolaridade materna foi classificada em três níveis: básico - até ao sexto ano; médio - do sétimo ao $12^{\circ}$ ano e superior - licenciatura, mestrado ou doutoramento.

Os dados demográficos, a utilização de corticoides pré-natais e a morbilidade perinatal foram analisados e comparados nos dois grupos: GUF versus GM. Considerou-se displasia broncopulmonar (DBP), caso o RN apresentasse necessidade de oxigénio suplementar às 36 semanas de idade pós-menstrual; sépsis tardia, como a ocorrência de infeção após as 72 horas de vida; enterocolite necrosante (NEC) classificada de acordo com os critérios de Bell. ${ }^{16}$ Considerou-se a persistência do canal arterial (PCA) por diagnóstico ecocardiográfico realizado por um cardiologista experiente, sempre que necessitasse de tratamento. Foi ainda comparada entre os grupos a presença de doença das membranas hialinas (DMH) e de retinopatia da prematuridade (ROP) com grau superior ou igual a $3 .{ }^{17}$

Foram consideradas lesões cerebrais graves a hemorragia peri e intraventricular (HPIV) de grau igual ou superior 
a três (classificação de Papile) ${ }^{18}$ e a leucomalácia quística periventricular (LQPV). ${ }^{19} \mathrm{~A}$ avaliação seriada com ecografia cerebral foi realizada de acordo com o consenso da Sociedade Portuguesa de Neonatologia (primeiros três dias e sétimo dia de vida, seguidos de avaliações semanais (na presença de alterações) ou quinzenais (em caso de exame normal), até às 40 semanas de idade pós-menstrual. ${ }^{20}$

$\mathrm{Na}$ consulta de seguimento da MBB, aos 24 meses de IC, foi aplicada, por duas educadoras do serviço com treino específico, a escala de avaliação de desenvolvimento psicomotor global denominada Schedule of Growing SkiIIs II. Através desta escala são avaliadas competências de desenvolvimento infantil, nas seguintes áreas: locomotora, manipulativa, visual, audição e linguagem, fala e linguagem, interação social, autonomia e cognitiva. Considerou-se um quociente de desenvolvimento global (QDG) normal o valor de 100 e desvio padrão de 15, calculado pelo quociente entre os resultados individuais alcançados e o valor médio considerado para a idade corrigida. Foi considerada alteração moderada a grave do neurodesenvolvimento, quando os resultados foram inferiores a $70,{ }^{21,22}$ o que corresponde a um atraso superior a dois desvio padrão abaixo da média para a idade corrigida.

O diagnóstico de PC foi estabelecido de acordo com a classificação internacional, da Surveillance of Cerebral
Palsy in Europe (SCPE) ${ }^{21}$ e foi confirmado pela observação das crianças entre os quatro e os cinco anos de idade por neuropediatra experiente. Existe um protocolo de colaboração entre a nossa instituição e o centro de paralisia cerebral local para onde foram referenciadas todas as crianças que apresentaram alterações motoras. Este centro confirma o diagnóstico, fornece o suporte necessário a estas situações e comunica regularmente a informação dos casos referenciados.

As acuidades auditiva e visual foram avaliadas de um modo sistemático.

Na primeira análise comparámos os grupos GUF e GM relativamente aos cofatores potencialmente influenciadores nas aquisições das competências do neurodesenvolvimento: IG, PN, administração de corticoides pré-natais, escolaridade materna, morbilidade neonatal, presença de lesões cerebrais graves (HPIV grau $\geq 3$ ou LQPV), alterações moderadas a graves do DPM (QDG e subáreas) e presença de PC.

$\mathrm{Na}$ segunda parte, o grupo de crianças nascidas de GUF foi comparado com os dois subgrupos de GM: GM-BC e GM-MC, de modo a avaliar o efeito da corionicidade.

\section{Análise estatística}

A análise estatística foi realizada com SPSS versão

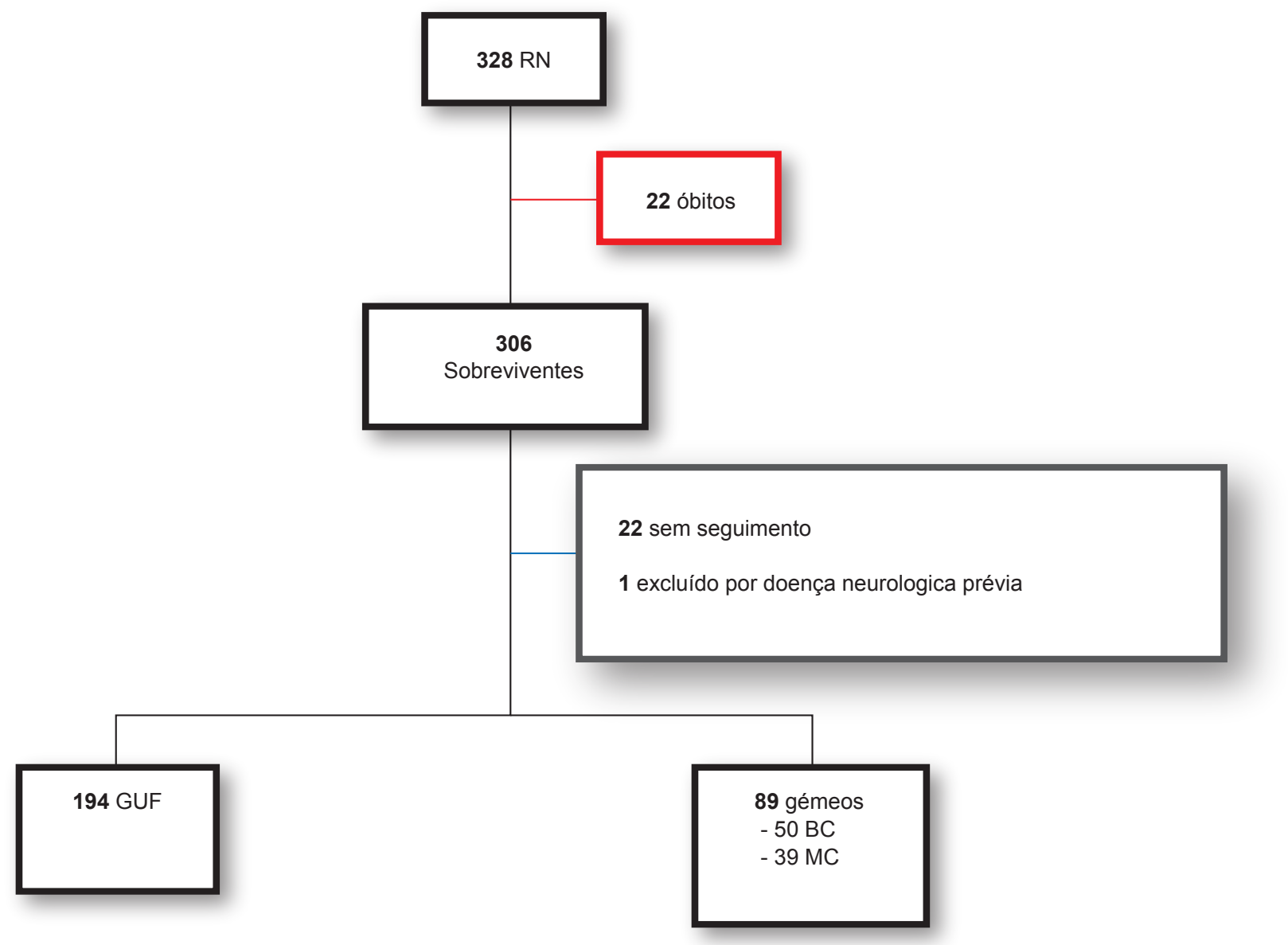

Figura 1 - População de estudo, dividida em dois grupos: recém-nascidos de gestação unifetal (GUF) e Gémeos sendo estes subdivididos em dois subgrupos: bicoriónicos (BC) e monocoriónicos (MC) 
20.0. Usou-se o teste $t$ de Student para comparação de médias, das variáveis contínuas, entre dois grupos não emparelhados. Utilizou-se o Qui quadrado (ou teste de Fisher) para análise das variáveis categóricas. Nas variáveis com significado estatístico realizou-se o ajustamento pela regressão logística e odds ratio para avaliar a relação da gemelaridade ou corionicidade com os outcomes estudados. Foi considerado um valor de $p<0,05$ (two tails) para indicar diferenças com significado estatístico.

\section{RESULTADOS}

No período de estudo a taxa de mortalidade foi de 22/328 RN (6,0\%). Dos 306 sobreviventes foram avaliadas 283 (91\%) crianças (Fig. 1). A população de estudo foi dividida em dois grupos: GUF ( $n=194)$ e GM $(n=89)$.

A média da idade materna não se revelou diferente em ambos os grupos, assim como a escolaridade materna. A comparação dos dois grupos (GUF versus $\mathrm{GM}$ ) em relação à IG, PN e à morbilidade precoce ou intervenções que poderiam estar associadas a alterações do neurodesenvolvimento (Tabela 1), revelou que o PN foi significativamente superior nos gémeos, contrariamente à utilização de corticoides pré-natais, que foi superior no grupo GUF.

A presença de lesões cerebrais graves, HPIV grau $\geq 3$ ou LQPV, não se revelou significativamente diferente entre os dois grupos.

A informação dos resultados da avaliação do neurodesenvolvimento foi obtida em 283 crianças.

O valor da média do QDG nas crianças de GUF foi de 95,2 \pm 10 e de 95,8 \pm 13 , no grupo GM, não mostrando diferenças significativas, contudo os resultados foram consideravelmente diferentes quando avaliámos a presença de alterações moderadas a graves do DPM.
As crianças que nasceram de GM apresentaram significativamente mais alterações moderadas a graves a nível do DPM nas subáreas locomoção, audição e linguagem, fala e linguagem e autonomia (Tabela 2), relativamente às nascidas de GUF.

Após ajustamento, por regressão logística, para os fatores: IG, PN e corticoides pré-natais, a gemelaridade manteve taxas significativamente superiores de alterações moderadas a graves nas subáreas da locomoção (OR ajustado 5,6), audição-linguagem (OR ajustado 5,4) e fala-linguagem (OR ajustado 4,1).

A surdez neurosensorial profunda com necessidade de implante coclear ocorreu num caso $(0,5 \%)$ do grupo GUF e em dois casos $(2,2 \%)$ de GM $(p=0,18)$ e a cegueira surgiu num caso do grupo GM.

A PC ocorreu em 4,1\% das crianças do GUF e em 6,7\% da GM, embora esta diferença não se tenha revelado significativa. No grupo GUF, das oito crianças com PC (sete tipo espástica bilateral e uma disquinética), duas (25.0\%) apresentaram compromisso funcional grave. No grupo de GM que apresentou seis crianças com PC (cinco tipo espástica bilateral e uma unilateral), o compromisso funcional grave estava presente em $50 \%$ dos casos.

Os subgrupos GM-BC ( $n=50)$ e GM-MC ( $n=39)$ foram comparados relativamente às alterações do neurodesenvolvimento incluindo a presença ou ausência de PC, com o grupo GUF e investigámos os fatores potencialmente influenciadores destes outcomes.

A idade e a escolaridade das mães não apresentaram diferenças significativas entre os grupos (Tabela 3), assim como a IG. O PN do subgrupo GM-MC foi significativamente superior ao do grupo GUF $(p=0,04)$.

$\mathrm{Na}$ comparação da morbilidade do período neonatal

Tabela 1 - Morbilidade neonatal (RN com IG inferior a 32 semanas e / ou PN < $1500 \mathrm{~g}$ ) dividida em dois grupos: recém nascidos de gestação unifetal (GUF)/ gémeos (GM)

\begin{tabular}{lccc}
\hline & $\begin{array}{c}\text { GUF } n(\%) \\
\mathrm{N}=194\end{array}$ & $\begin{array}{c}\text { GM } n(\%) \\
\mathrm{N}=89\end{array}$ & $\mathrm{p}$ OR (IC* 95\%) \\
\hline IG (semanas) - média (DP) & $29,5( \pm 2,1)$ & $30( \pm 2,2)$ & 0,06 \\
PN (g) - média (DP) & $1217( \pm 313)$ & $1297( \pm 324)$ & 0,03 \\
Género & & & \\
$\quad$ Masculino & $113(58,2)$ & $45(50,6)$ & 0,16 \\
$\quad$ Feminino & $81(41,8)$ & $44(49,4)$ & $0,46[0,22-0,9]$ \\
Corticoides pré natais & $175(90,2)$ & $72(80,9)$ & 0,45 \\
Reanimação com TET & $68(34,9)$ & $27(30,3)$ & 0,69 \\
DMH & $116(59,8)$ & $51(57,3)$ & 0,78 \\
PCA tratado & $31(15,9)$ & $13(14,6)$ & 0,97 \\
Sepsis tardia & $42(21,5)$ & $19(21,3)$ & 0,66 \\
NEC grau $\geq 2$ & $4(2,2)$ & $1(1,1)$ & 0,75 \\
DBP & $9(4,6)$ & $3(3,4)$ & 0,68 \\
ROP grau $\geq 3$ & $4(2,2)$ & $1(1,2)$ & 0.31 \\
HPIV grau $\geq 3$ & $6(3,1)$ & $5(5,6)$ & 0,29 \\
LQPV & $5(2,6)$ & $5(5,6)$ & \\
\hline
\end{tabular}

IG: Idade gestacional; PN: Peso de nascimento; DMH: Doença das membranas hialinas; PCA: Persistência do canal arterial; NEC: Enterocolite necrosante; DBP: Displasia broncopulmonar; ROP: Retinopatia da prematuridade; HPIV: Hemorragia peri e intraventricular; LQPV: Leucomalácia quística peri e intraventricular; IC*: Intervalo de confiança 
Tabela 2 - Avaliação aos 24 meses: alterações moderadas a graves nas várias áreas do desenvolvimento psicomotor (DPM) e paralisia cerebral, nas crianças com IG inferior a 32 semanas e/ou peso de nascimento $(\mathrm{PN})<1500 \mathrm{~g}$

\begin{tabular}{|c|c|c|c|c|}
\hline & $\begin{array}{c}\text { GUF } n(\%) \\
\quad N=194\end{array}$ & $\begin{array}{c}\text { GM } n(\%) \\
N=89\end{array}$ & $p$ & OR $\left(I^{* *} 95 \%\right)$ \\
\hline QD global & $9(4,6)$ & $8(9,0)$ & 0,15 & \\
\hline Locomoção* & $2(1,3)$ & $6(8,0)$ & 0,017 & $6,4[1,2-32,9]$ \\
\hline Manipulação* & $5(3,3)$ & $5(6,6)$ & 0,30 & \\
\hline Visão* & $3(2,0)$ & $3(4,0)$ & 0,40 & \\
\hline Audição e linguagem* & $3(2,0)$ & $7(9,3)$ & 0,017 & $5,0[1,2-20,2]$ \\
\hline Fala e linguagem* & $6(3,1)$ & $10(13,3)$ & 0,014 & $3,7[1,3-10,6]$ \\
\hline Interação social* & $2(1,3)$ & $3(4,0)$ & 0,33 & \\
\hline Autonomia* & $2(1,3)$ & $5(6,6)$ & 0,042 & $5,3[1,0-28,1]$ \\
\hline Cognição* & $4(2,6)$ & $4(5,3)$ & 0,44 & \\
\hline Paralisia cerebral & $8(4,1)$ & $6(6,7)$ & 0,38 & \\
\hline
\end{tabular}

GUF: Gestação unifetal; GM: Gémeos; QD: Quociente do desenvolvimento

*As subáreas do QD referem-se a um total de 225:150 GFU e 75 gémeos; IC**: Intervalo de confiança

Tabela 3 - Caraterísticas demográficas da população estudada, comparando o grupo de gestação unifetal (GUF) com gémeos bicorióni$\cos (\mathrm{GM}-\mathrm{BC})$ e monocoriónicos (GM - MC)

\begin{tabular}{|c|c|c|c|c|}
\hline & $\begin{array}{l}\text { GUF n (\%) } \\
\quad N=194\end{array}$ & $\begin{array}{c}\mathrm{GM}-\mathrm{BC} \mathrm{n}(\%) \\
\mathrm{N}=25\end{array}$ & $\begin{array}{c}\text { GM }- \text { MC } n(\%) \\
N=20\end{array}$ & $p$ \\
\hline Idade materna (anos) mediana ( $\min -\max$ ) & $30(16-43)$ & $31(22-42)$ & $31,5(21-41)$ & 0,052 \\
\hline $\begin{array}{l}\text { Nível escolaridade materna } \\
\mathrm{N}=168 / 239(60 \%)^{\star *} \\
\text { Básica } \\
\text { Média } \\
\text { Superior }\end{array}$ & $\begin{array}{l}N=134 \\
25(18,7) \\
72(53,7) \\
37(27,6)\end{array}$ & $\begin{array}{c}N=19 \\
1(5,2) \\
8(42,2) \\
10(52,6)\end{array}$ & $\begin{array}{l}N=15 \\
2(13,4) \\
8(53,3) \\
5(33,3)\end{array}$ & 0,95 \\
\hline & $\begin{array}{c}\text { RN GUF n (\%) } \\
\quad N=194\end{array}$ & $\begin{array}{c}\mathbf{G M}-\mathbf{B C} n(\%) \\
N=50\end{array}$ & $\begin{array}{c}\text { GM - MC n (\%) } \\
N=39\end{array}$ & \\
\hline Idade gestacional (semanas) média (DP) & $29,5( \pm 2,1)$ & $29,9( \pm 2,2)$ & $30,2( \pm 2,2)$ & 0,057 \\
\hline Peso de nascimento (g), média (DP) & $1217( \pm 313)$ & $1285( \pm 301)$ & $1312( \pm 355)$ & $0,041^{*}$ \\
\hline $\begin{array}{l}\text { Distribuição por sexo } \\
\text { Masculino } \\
\text { Feminino }\end{array}$ & $\begin{array}{l}113(58,2) \\
81(41,8)\end{array}$ & $\begin{array}{l}27(54,0) \\
23(46,0)\end{array}$ & $\begin{array}{l}17(43,6) \\
22(56,4)\end{array}$ & 0,09 \\
\hline
\end{tabular}

* Resultado da comparação do grupo monocoriónico com o grupo de RN unifetal; ** Resultado obtido em 168 das 239 mães (60,0\%).

não encontrámos diferenças com significado estatístico entre o grupo GUF e qualquer dos subgrupos (GM-MC, GM$-B C)$.

A avaliação das lesões cerebrais revelou que os RN do subgrupo GM-MC sofriam significativamente de mais lesões de LQPV $(12,8 \%)$ do que os RN do grupo GUF $(2,6 \%)(p=0,01$; OR: 5,$5 ;$ IC:1,5 - 20,2). Esta diferença não foi encontrada nos $R N$ do subgrupo GM-BC, quando comparados com os RN de GUF, o mesmo se verificou em relação às alterações moderadas a graves do neurodesenvolvimento e à presença de PC (Tabela 4).

Quando comparadas as crianças do grupo GUF com as do subgrupo GM-MC verificaram-se diferenças significativas quer em relação às alterações moderadas e graves do DPM, quer em relação à presença de PC revelando este subgrupo taxas superiores de sequelas (Tabela 5 ).

Através do ajustamento por regressão logística para os fatores que revelaram diferença com significado estatístico (PN, LQPV), verificámos que o subgrupo GM-MC revelou risco independente de alterações moderadas a graves do DPM global e nas subáreas da locomoção (OR ajustado 12,2), audição-linguagem ( OR ajustado 6,5), fala-linguagem (OR ajustado 5,4$)$ e autonomia (OR ajustado 7,2 ).

A PC, que ocorreu em $6 / 39(15,4 \%)$ das crianças do subgrupo GM-MC foi significativamente superior em relação ao grupo GUF (4,0\%), o que também foi suportado pela análise por regressão logística (OR ajustado 4,2). Dois dos seis casos de PC ocorreram em crianças em que houve morte fetal do co-gémeo (um destes teve simultaneamente STFF).

A LQPV associou-se também a maior risco de PC (OR ajustado 28,1).

Os RN do subgrupo GM-MC revelaram taxas significativamente mais elevadas de LPVQ $(p=0,01), \operatorname{PC}(p=0,01)$ e alterações do DPM nas áreas da locomoção $(p=0,01)$, em relação aos $\mathrm{RN}$ do subgrupo GM-BC. Neste subgrupo a formação académica universitária das mães foi superior à do subgrupo dos gémeos $M C(p=0,03)$. 
Tabela 4 - Alterações moderadas a graves do desenvolvimento psicomotor e presença de paralisia cerebral, nos grupos de gestação unifetal versus gémeos bicoriónicos

\begin{tabular}{|c|c|c|c|}
\hline & $\begin{array}{c}\text { RN GUF n (\%) } \\
\quad \mathrm{N}=194\end{array}$ & $\begin{array}{c}\mathbf{G M}-\mathbf{B C} n(\%) \\
N=50\end{array}$ & $p$ \\
\hline $\mathrm{N}^{\circ}$ de $\mathrm{RN}$ & 194 & 50 & \\
\hline QDG & $9(4,6)$ & $2(4,0)$ & 0,60 \\
\hline Locomoção $^{a}$ & $2(1,3)$ & $0(0,0)$ & 0,60 \\
\hline Manipulação a & $5(3,3)$ & $1(2,4)$ & 0,60 \\
\hline Visão $^{a}$ & $3(2,0)$ & $0(0,0)$ & 0,47 \\
\hline Audição e linguagem ${ }^{a}$ & $3(2,0)$ & $2(4,8)$ & 0,30 \\
\hline Fala e linguagem ${ }^{a}$ & $6(3,1)$ & $3(7,1)$ & 0.31 \\
\hline Interação social ${ }^{a}$ & $2(1,3)$ & $0(0,0)$ & 0,60 \\
\hline Autonomia $^{a}$ & $2(1,3)$ & $1(2,4)$ & 0,52 \\
\hline Cognição $^{a}$ & $4(2,6)$ & $0(0,0)$ & 0,36 \\
\hline Paralisia cerebral & $8(4,1)$ & $0(0,0)$ & 0,15 \\
\hline
\end{tabular}

QDG: Quociente de desenvolvimento global; RN GUF: Recém-nascidos de gestação unifetal; GM - BC: Gémeos bicoriónicos; n: Número de crianças com alterações moderadas a graves, a nível das áreas do desenvolvimento psicomotor e paralisia cerebral; a: As subáreas do quociente de desenvolvimento referem-se a um total de 225, sendo150 de gestação unifetal e 75 gémeos, dos quais 42 bicoriónicos.

Tabela 5 - Alterações moderadas a graves do desenvolvimento psicomotor e presença de paralisia cerebral, nos grupos de gestação unifetal versus gémeos monocoriónicos

\begin{tabular}{|c|c|c|c|c|c|}
\hline & $\begin{array}{c}\text { RN GUF n (\%) } \\
\quad N=194\end{array}$ & $\begin{array}{c}\text { GM - MC n (\%) } \\
N=39\end{array}$ & $p$ & OR (IC* 95\%) & OR ajustado (IC* 95\%) \\
\hline QDG & $9(4,6)$ & $6(15,4)$ & 0,03 & $3,6[1,2-10,8]$ & $3,6[1,1-12,9]$ \\
\hline Locomoção $^{a}$ & $2(1,3)$ & $6(18,0)$ & $<0,01$ & $16,4[3,1-85,8]$ & $12,2[2,3-71,2]$ \\
\hline Manipulação ${ }^{a}$ & $5(3,3)$ & $4(12,1)$ & 0,06 & & \\
\hline Visão ${ }^{a}$ & $3(2,0)$ & $3(9,1)$ & 0,07 & & \\
\hline Audição e linguagem ${ }^{\text {a }}$ & $3(2,0)$ & $5(15,2)$ & 0,01 & $8,7[1,9-38,7]$ & $6,5[1,3-31,3]$ \\
\hline Fala e linguagem ${ }^{a}$ & $6(3,1)$ & $7(21,2)$ & 0,01 & $6,4[2,0-20,7]$ & $5,4[1,6-18,4]$ \\
\hline Interação social ${ }^{\mathrm{a}}$ & $2(1,3)$ & $3(9,1)$ & 0,04 & $7,4[1,1-46,2]$ & \\
\hline Autonomia $^{a}$ & $2(1,3)$ & $4(12,1)$ & 0,01 & $10,2[1,7-58,3]$ & $7,2[1,1-43,5]$ \\
\hline Cognição ${ }^{a}$ & $4(2,6)$ & $4(12,1)$ & 0,04 & $5,0[1,1-21,2]$ & \\
\hline Paralisia cerebral & $8(4,1)$ & $6(15,4)$ & 0,02 & $4,2[1,3-12,9]$ & $4,2[1,1-13,2]$ \\
\hline
\end{tabular}

QDG: Quociente de desenvolvimento global; RNGUF: Recém-nascidos de gestação unifetal; GM - MC: Gémeos monocoriónicos; n: Número de crianças com alterações moderadas a graves, a nível das áreas do desenvolvimento psicomotor e paralisia cerebral; : As subáreas do quociente de desenvolvimento referem-se a um total de 225 , sendo150 de gestação unifetal e 75 gémeos, dos quais 33 monocoriónicos.

\section{DISCUSSÃO}

A preocupação com as alterações do neurodesenvolvimento em gémeos tem sido evidente na literatura desde há várias décadas. ${ }^{3-5,9}$

No presente estudo quando se comparou a frequência de lesões cerebrais, detetadas por ecografias cerebrais seriadas, em crianças nascidas de GUF com GM não se encontrou diferença com significado estatístico. Contudo, a taxa de LQPV foi significativamente mais elevada nos gémeos $\mathrm{MC}(12,8 \%)$ relativamente à das crianças que nasceram de GUF $(2,6 \%)$, traduzindo-se num risco de ocorrência cinco vezes superior.

Estes resultados estão de acordo com a literatura que refere maior incidência de neuromorbilidade, detetada por neurosonografia, em gémeos monocoriónicos quando comparados com os bicoriónicos ou com RN de gestação única. ${ }^{5}$
Este estudo obteve avaliação objetiva do DPM aos 24 meses e informação sobre a presença de $\mathrm{PC}$ entre os quatro e os cinco anos numa elevada fração da população inicial $(91,0 \%)$. Noutros estudos, as taxas de vigilância são muito variáveis. Uma revisão sistemática recente refere percentagens de vigilância entre 71 e $100 \%{ }^{24}$ Considera-se ideal que esta taxa seja superior a $90,0 \%$ da população inicial, ${ }^{11}$ o que foi obtido no nosso trabalho sendo, por isso, um dos seus pontos fortes.

No nosso estudo, ao compararmos o grupo de gestação unifetal com o grupo total dos gémeos (sem divisão pelo tipo de corionicidade) evidenciamos que ser gémeo está associado a taxas quatro a seis vezes mais elevadas de alterações do neurodesenvolvimento, especificamente de défices nas áreas de locomoção, compreensão e expressão da linguagem e autonomia, relativamente a crianças que nasceram de gestações unifetais. Após ajustamento, 
por regressão logística, para a IG, PN e administração de corticoides pré-natais verificou-se que esta diferença se manteve, particularmente nas áreas de locomoção e linguagem. Vários trabalhos descrevem a gemelaridade, como fator de risco para estas alterações. ${ }^{1,3}$

Quando foi comparado o GUF com os subtipos de corionicidade dos gémeos só foram evidenciadas diferenças significativas com os monocoriónicos, quer em relação à alteração moderada a grave do quociente de desenvolvimento global (4,6\% no grupo GUF versus $15,4 \%$ no GM-MC, com risco quatro vezes superior de atraso global do desenvolvimento psicomotor nos gémeos $\mathrm{MC}$ ), quer em relação aos défices verificados na maioria das subáreas do desenvolvimento psicomotor global.

As alterações graves nalgumas subáreas, sobretudo da locomoção, eram esperadas, atendendo a que corresponderam na sua totalidade às crianças que vieram a ser diagnosticados, mais tarde, com paralisia cerebral. O atraso na aquisição de competências de outras subáreas do desenvolvimento como a linguagem compreensiva $(15,2 \%)$, expressiva $(21,2 \%)$ e a autonomia $(12,1 \%)$ representou riscos, respetivamente de seis, cinco e sete vezes superiores, nas crianças que nasceram de GM-MC em relação às que nasceram de GUF.

A consistência destas alterações e a ausência destas diferenças em relação aos gémeos bicoriónicos realça o papel da corionicidade na base das sequelas do neurodesenvolvimento. Resultados semelhantes foram encontrados por Adegbite et $a^{25}$ que demonstraram alterações do neurodesenvolvimento em $15,0 \%$ dos gémeos monocoriónicos contra 3,0\% nos bicoriónicos. Contudo, os resultados publicados revelam taxas de alterações do neurodesenvolvimento muito variáveis ${ }^{2,3,26}$ e por vezes sem controlo das variáveis confundentes. Um trabalho recente,${ }^{27}$ tratando a corionicidade, não revelou uma frequência superior de alterações de neurodesenvolvimento nos gémeos monocoriónicos em relação aos bicoriónicos, tendo demonstrado que a prematuridade representou um fator de risco independente para aquelas alterações. A prematuridade tem sido referida como fator de risco para o desenvolvimento de sequelas neurológicas na maior parte da literatura, daí a necessidade de estratificar este tipo de estudos em relação à idade gestacional.

A ausência da informação sobre a corionicidade limita, também, as conclusões nalguns estudos que comparam gémeos, divididos por género: concordante ou discordante..$^{5,12}$

O nosso trabalho, realizado numa população de grandes prematuros, comparou grupos estratificados em relação à idade gestacional e à corionicidade, nas gestações gemelares, permitindo realizar a análise, de acordo com ambas as variáveis, o que poderá representar um ponto forte deste estudo.

Apesar da taxa de PC nos gémeos $(6,7 \%)$ ter sido superior à das crianças de GUF $(4,1 \%)$, esta diferença não foi significativa. Quando essa análise foi realizada em relação aos gémeos monocoriónicos a taxa de $\mathrm{PC}$ revelou-se muito mais elevada, (15,4\%), com significado estatístico, o que não aconteceu no grupo de gémeos bicoriónicos.

Dois casos de PC em crianças com óbito do co-gémeo (sendo um destes simultaneamente STFF), alertam para um possível papel das complicações da corionicidade para esta sequela neurológica.

Estes dados replicam os resultados de outros trabalhos e reforçam o que tem vindo a ser defendido, que a responsabilidade pelo aumento da taxa de $\mathrm{PC}$ em gémeos está relacionada com a corionicidade e as complicações que the são inerentes. ${ }^{2,5,9,28-34}$

De facto, Pharoah et al ${ }^{12}$ atribuem um risco de alterações neurológicas graves a cerca de metade dos sobreviventes de uma gestação com óbito do co-gémeo. $\mathrm{Na}$ revisão sistemática de Ong et a/ ${ }^{11}$ é referido um risco de alterações neurológicas em $18,0 \%$ dos sobreviventes de gestações com óbito de um gémeo, representando um risco de seis e quatro vezes superior nas gestações monocoriónicas e bicoriónicas, respetivamente.

A causa destas alterações associadas à morte fetal de um gémeo tem sido explicada pela passagem de material trombótico do feto morto para o vivo com consequentes alterações da cascata da coagulação ou por alterações hemodinâmicas após a morte do feto, com transfusão do feto vivo para o morto. $4,10,11,13,14$

A LQPV tendo-se associado significativamente aos gémeos monocoriónicos e revelado factor de risco independente para a PC, poderá ter dado algum contributo para a ocorrência desta neuromorbibilidade. Desde há cerca de duas décadas, deVries ${ }^{35}$ referiu a relação entre a existência de LQPV e a ocorrência de paralisia cerebral, salientando a importância da ressonância magnética cerebral, a qual pode detetar alterações ocorridas no período peri-natal em casos em que a ecografia cerebral foi normal. Um estudo nacional, ${ }^{36}$ do grupo de registo nacional de muito baixo peso, relacionou a PC com as idades gestacionais mais baixas e caracterizou a LQPV como uma variável com influência significativa na ocorrência da paralisia cerebral.

Este trabalho, realizado em gémeos grandes prematuros, com uma elevada taxa de vigilância longitudinal e adequado conhecimento da corionicidade contribui para o conhecimento da neuromorbilidade em crianças nascidas de gestações múltiplas.

É de salientar como limitações deste estudo tratar-se de uma análise retrospectiva, embora o registo sistemático e estruturado dos dados, em contexto do registo nacional de MBP permita a sua uniformização bem como a redução da taxa de casos omissos.

A avaliação do neurodesenvolvimento aos 24 meses e recorrendo a testes considerados de rastreio como é o caso da escala Growing Skills serão fatores limitantes para o diagnóstico de défices ligeiros das competências de linguagem, de interação social e da capacidade cognitiva.

O seguimento destas crianças até à idade escolar seria desejável, pois só neste período etário é possível excluir problemas relacionados com o comportamento disruptivo, a atenção, a memória e a função executiva, nas crianças 
que, até aos dois anos ainda não evidenciaram problemas de neurodesenvolvimento.

\section{CONCLUSÃO}

Este trabalho permite concluir que os gémeos monocoriónicos apresentaram um risco muito superior de sofrer de alterações graves do neurodesenvolvimento, incluindo a PC, quando comparados com crianças nascidas de gravidez unifetal com semelhante idade gestacional. A previsão destas sequelas permite informar adequadamente as famílias e estabelecer programas de vigilância e de apoio mais adequados, às crianças nascidas de gravidez múltipla, sobretudo se for monocoriónica. Sugere-se por isso a sua vigilância em consulta específica de neurodesenvolvimento, com apoio de equipa multidisciplinar até à idade escolar, podendo assim dar resposta educacional e terapêutica atempada às suas eventuais dificuldades motoras, comportamentais e de aprendizagem.

Futuros estudos são necessários para avaliar, nas gestações gemelares monocoriónicas, a efetiva contribuição da corionicidade e das suas complicações para as referidas alterações do neurodesenvolvimento.

\section{REFERÊNCIAS}

1. Wadhawan R, Oh W, Perritt R, McDonald S, Das A, Poole W, et al. Twin gestation and neurodevelopmental outcome in extremely low birth weight infants. Pediatrics. 2009;123:e220-7.

2. Hack K, Derks J, Elias S, Franx A, Roos E, Voerman S, et al. Increased perinatal mortality and morbidity in monochorionic versus dichorionic twin pregnancies: clinical implications of a large Duttch cohort study. BJOG. 2008;115:58-67.

3. Lorenz J. Neurodevelopmental outcomes of twins. Sem Perinatol. 2012;36:201-12.

4. Pharoah PO. Neurological outcome in twins. Semin Neonatol. 2002;7:223-30.

5. Adegbite A, Castille S, Ward S, Bajoria R. Neuromorbidity in preterm twins in relation to chorionicity and discordant birth weight. Am J Obstet Gynecol. 2004;190:15663.

6. Andrada M, Virella D, Calado E, Gouveia R, Folha T. Paralisia cerebral aos 5 anos de idade em Portugal. Monografia. Lisboa: Federação das Associações Portuguesas de Paralisia Cerebral; 2009.

7. Himmelmann K, Hagberg G, Uvebrant P. The changing panorama of cerebral palsy in Sweden. Prevalence and origin in the birth-year period 1999-2002. Acta Paediatr. 2010;99:1337-43.

8. Oskoui M, Coutinho F, Dykeman J, Jetté N, Pringshein T. An update on the prevalence of cerebral palsy: a systematic review and metaanalysis. Dev Med Child Neurol. 2013;55:509-19.

9. Karien H, Koopman-Esseboom C, Derks J, Elias S, Kleine M, Baerts $\mathrm{W}$, et al. Long-term neurodevelopmental outcome of monochorionic and matched dichorionic twins. PLoS One. 2009;4:e6815.

10. Fortin A, Rajguru M, Madelenat P, Mahieu-Caputo D. Pronostic neurologique des enfants issus de grossesse gémellaire. Gynecol Obstet Fertil. 2005;33:563-9.

11. Ong SS, Zamora J. Khan KS, Kilby MD. Prognosis for the co-twin following single-twin death: a systematic review. BJOG. 2006;113:9928.

12. Pharoah PO. Consequences of in-utero death in a twin pregnancy. Lancet. 2000;355:1597-602.

13. DebillonT. Mortalité et morbidité chez les jumeaux. Arch Pediatr. 2004;11:659-60.

14. Ancel P, Mazaubrun C, Bréart G. Grossesses multiples, lieu de naissance et mortalité des grands prématurés: premiers résultats d' EPIPAGE Ile de France. J Gynecol Obstet Biol Reprod. 2001;30:48-50.

15. Livinec F, Ancel P, Marret S. Prenatal risk factors for cerebral palsy in very preterm singletons and twins. Obstet Gynecol. 2005;105:1341-7.

16. Bell MJ, Ternberg JI, Feigin RD, Keating JP, Marshall R, Barton L, et al. Neonatal necrotizing enterocolitis: therapeutic decisions based on

\section{PROTECÇÃO DE PESSOAS E ANIMAIS}

Os autores declaram que os procedimentos seguidos estavam de acordo com os regulamentos estabelecidos pelos responsáveis da Comissão de Investigação Clínica e Ética e de acordo com a Declaração de Helsínquia da Associação Médica Mundial.

\section{CONFIDENCIALIDADE DOS DADOS}

Os autores declaram ter seguido os protocolos do seu centro de trabalho acerca da publicação de dados.

\section{CONFLITOS DE INTERESSE}

Os autores declaram não existirem conflitos de interesse relativamente ao presente artigo.

\section{FONTES DE FINANCIAMENTO}

Os autores declaram não ter recebido subsídios ou bolsas para a elaboração do artigo.

clinical staging. Ann Surg. 1978;187:1-7.

17. International Committee for the Classification of Retinopathy of Prematurity. The International Classification of Retinopathy of Prematurity revisited. Arch Ophthalmol. 2005;123:991-9.

18. Papile A, Burstein J, Burstein R, Koffler H. Incidence and evolution of subependymal and intraventricular hemorrhsge: A study of infants with birth weights less than 1500gm. J Pediatr. 1978;92:529-34.

19. De Vries LS, Eken P, Dubowitz LM. The spectrum of leukomalacia using cranial ultrasound. Behav Brain Res. 1992;49:1-6.

20. Taborda A, Pereira A, Graça A, Conceição C, Faria C, Trindade C, et al. Revisão do consenso de neuro-imagiologia neonatal - versão maio 2013. [Consultado 2015 set 26]. Disponível em: http://www. lusoneonatologia.com/site/upload/consensos/2010-Neuroimagiologia. pdf.

21. Larroque B, Ancel P, Marret S, Marchand L, André M, Arnaud C, et al. Neurodevelopmental disabilities and special care of 5-year-old children born before 33 weeks of gestation (the EPIPAGE study): a longitudinal cohort study. Lancet. 2008; 813-20.

22. Wood N, Marlow N, Costeloe, K, Chir B, Gibson A, Wilkinson A, for the EPICure Study Group. Neurologic and developmental disability after extremely preterm birth. N Engl J Med. 2000;343:378-84.

23. Rosenbaum P, Paneth N, Leviton A, Goldstein M, Bax M, Damiano D, et al. A report: the definition and classification of cerebral palsy. Dev Med Child Neurol. 2007;49:8-14.

24. Guillen U, DeMauro S, Zupancic J, Roberts R, Schmidt B, Kirpalani $\mathrm{H}$. Relationship between attrition and neurodevelopmental impairment rates in extremely preterm infants at 18 to 24 months. A systematic review. Arch Pediatr Adolesc Med. 2012;166:178-84.

25. Adegbite A, Castille S, Ward S. Neuromorbidity in preterm twins in relation to chorionicity and discordant birth weight. Am J Obstet Gynecol. 2004;190:156-63.

26. Leonard C, Piecuch R. Outcome of very low birth weight: Multiple gestation versus singletons. Pediatrics. 1994;93:611-5.

27. Kawamura H, Ishii K, Yonetani N, Mabuchi A, Hayashi S, Mitsuda N. Significance of chorionicity on long-term outcome of low birthweight infants of $<1500 \mathrm{~g}$ in twin pregnancies. J Obstet Gynaecol Res. 2015;41:1185-92.

28. Lopriore E, Nagel H, Vandenbussche F, Walther F. Long-term neurodevelopmental outcome in twin-to twin transfusion syndrome. Am J Obstet Gynecol. 2003;189:1314-9.

29. Burguet A, Monnet E, Pauchard J. Some risk factors for cerebral palsy in very preterm infants: importance of premature rupture of membranes and monochorionic twin placentation. Biol Neonate. 1999;75:177-86. 
30. Pharoah PO, Dundar Y. Monozygotic twinning, cerebral palsy and congenital anomalies. Hum Reprod Update. 2009;15:639-48.

31. Williams K, Hennesay E, Alberman E. Cerebral palsy: effects of twinning, birth weight and gestational age. Arch Dis Child. 1996;75:F178-82.

32. Forrester KR, Keegan KM, Schmidt JW. Neurological impairment in a surviving twin following intrauterine fetal demise of the co-twin: a case study. J Neonatal Perinatal Med. 2013;6:83-88.

33. van Klink J, Koopman HM, Middeldorp JM, Klumper FJ, Rijken M, Oepkes $\mathrm{D}$, et al. Long-term neurodevelopmental outcome after selective feticide in monochorionic pregnancies. BJOG. 2015;122:1517-24.

34. Fortin A, Rajguru M, Madelenat P, Mahieu-Caputo D. Pronostic neurologique des enfants issus de grossesse gémellaire. Neurological outcome of children from twin pregnancies. Gynecol Obstet Fertil. 2005;33:563-9.

35. De Vries LS, Eken P, Groenendaal F, van Haastert IC, Meiners LC. Correlation between the degree of perivenricular leukomalacia disgnosed using cranial ultrasound and MRI later in infancy in children with cerebral palsy. Neuropediatrics. 1993;24:263-8.

36. Cunha M, Bettencourt A, Almeida A, Mimoso G, Soares P, Tomé T, et al. O recém-nascido de extremo baixo peso. Estado aos 2-3 anos. Resultados do Registo Nacional de Muito Baixo Peso de 2005 e 2006. Acta Pediatr Port. 2013;44:1-8. 